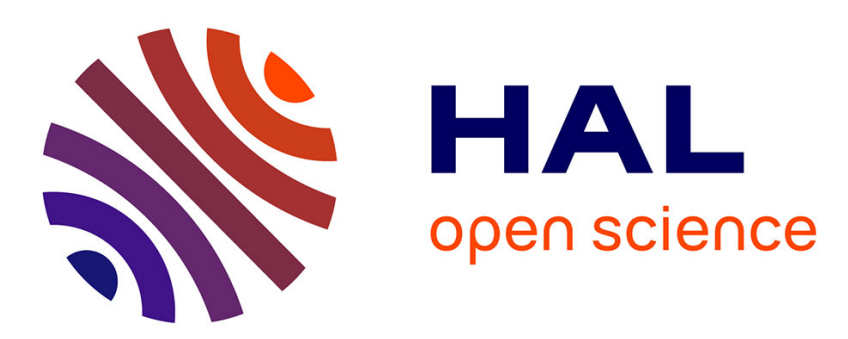

\title{
Note sur la présence de lames aménagées par technique de Kostienki dans les couches gravettiennes du Blot (Cerzat,Haute-Loire).
}

Laurent Klaric

\section{- To cite this version:}

Laurent Klaric. Note sur la présence de lames aménagées par technique de Kostienki dans les couches gravettiennes du Blot (Cerzat,Haute-Loire).. Bulletin de la Société préhistorique française, 2000, 97 (4), pp.625-636. 10.3406/bspf.2000.11169 . halshs-00366351

\section{HAL Id: halshs-00366351 \\ https://shs.hal.science/halshs-00366351}

Submitted on 6 Mar 2009

HAL is a multi-disciplinary open access archive for the deposit and dissemination of scientific research documents, whether they are published or not. The documents may come from teaching and research institutions in France or abroad, or from public or private research centers.
L'archive ouverte pluridisciplinaire HAL, est destinée au dépôt et à la diffusion de documents scientifiques de niveau recherche, publiés ou non, émanant des établissements d'enseignement et de recherche français ou étrangers, des laboratoires publics ou privés. 


\title{
Note sur la présence de lames aménagées par technique de Kostienki dans les couches gravettiennes du Blot (Cerzat, Haute-Loire)
}

\begin{abstract}
Résumé
La présence inédite de lames aménagées par "technique de Kostienki" (ou couteaux de Kostienki) dans les couches gravettiennes de l'abri Blot a conduit à un nouvel examen de ces pièces. Sur la base d'une étude technologique approfondie, il a été démontré que de tels artefacts ont, en association ou en complément avec des pièces assimilées à des burins, fort bien pu jouer le rôle de nucléus à lamelles dans un contexte particulier de production d'armatures microlithiques. Le Blot est le second site en France à compter de tels artefacts, le premier étant Corbiac (Dordogne). Il s'agit donc de discuter leur fonction plus précisément dans le but de permettre leur identification dans d'autres contextes que le Gravettien d'Europe centrale et orientale.
\end{abstract}

\begin{abstract}
The unprecedented presence of Kostienki-technique prepared blades (also called Kostienki knives) in the Gravettian layers at Le Blot leads to a new analysis of these artefacts. Thorough technological studies have pointed to the possible role of these items as cores, in association with or complementary to burin-forms, in a particular context of backed-bladelet production. Le Blot is the second French site yielding such artefacts, the other being Corbiac (Dordogne). The aim is thus to provide some elements to characterise and discuss their function in order to allow their identification in contexts other than the Gravettian of Central and Eastern Europe.
\end{abstract}

\section{LES LAMES AMÉNAGÉES PAR TECHNIQUE DE KOSTIENKI}

\section{Rappel typologique}

Les lames aménagées par technique de Kostienki, ou "couteaux de Kostienki", étant des artefacts relativement peu répandus en Europe occidentale, il convient de faire ici un bref rappel de leurs principales caractéristiques typologiques. Sans reprendre la description complexe de V.I. Belayeva présentée par J. Kozlowski dans sa synthèse sur les pièces découvertes à Corbiac (Kozlowski, 1984, p. 37), nous reproduirons ici une définition plus courte de l'aménagement des lames par technique de Kostienki qui fut proposée par M. Otte: "Il s'agit d'une retouche inverse, généralement du côté proximal et à partir de laquelle des enlèvements lamellaires ont été débités sur la face supérieure. Elle peut être appliquée soit à la base de lames utilisées, soit à l'extrémité de lames retouchées, soit à l'opposé d'un 
autre outil. Quelques fois, une retouche inverse proximale ne présente pas d'enlèvements supérieurs mais se trouve dans les mêmes formes d'associations (préparation de couteau de Kostienki?). Dans d'autres cas, les enlèvements sont portés à partir d'une cassure proximale" (Otte, 1980, p. 54-55).

\section{Historique de la recherche}

Ces artefacts furent identifiés pour la première fois par l'abbé Breuil à Predmosti (fig. 1) en Tchécoslovaquie (Breuil, 1924). Il les considérait (sans pour autant les nommer) comme un type "trop fréquent pour ne pas être systématique"; type qu'il assimilait à "une sorte de gouge "(Breuil, 1924, p. 530). Aucun élément comparable n'avait encore jamais été rencontré dans les sites d'Europe occidentale. C'est P. Efimienko qui nomma ces pièces "couteaux de Kostienki" à partir de ses observations sur le matériel de Kostienki I (fig. 7, $n^{\circ} 3$ ) (Efimienko, 1958). Il interprétait alors les enlèvements qui se développaient sur la face supérieure (ou avers) comme une technique spéciale d'amincissement des lames en vue d'un emmanchement. D'un point de vue chrono-culturel ces pièces apparaissaient comme des marqueurs spécifiques du Gravettien de la Plaine russe ou "Kostienkien" (Efimienko, 1958).

En 1961, M.D. Gvozdover signala l'existence d'une assez grande série de couteaux de Kostienki sur le site d'Avdeevo: elle considérait ces pièces non pas comme un type d'outil particulier mais plutôt comme le résultat d'une technique spécifique. Cette dernière, définie comme caractéristique du Kostienkien, était alors considérée comme le moyen d'obtenir des outils assez différenciés et hétérogènes du point de vue morphologique et fonctionnel (Gvozdover, 1961). Cette appellation particulière de "couteaux de Kostienki" fut reprise par J. de Heinzelin pour désigner certaines pièces de Maisières-Canal qui s'avérèrent en fait plus proches de pièces esquillées (Heinzelin, 1962 et 1973). En effet, il ne semble pas que des pièces répondant à la définition précédente aient été présentes dans le Gravettien de Belgique (Otte, 1979). Il fallut attendre quelques années pour que d'autres couteaux de Kostienki, alors interprétés comme des "ciseaux" ou des "gouges", soient identifiés dans le Gravettien Morave ou "Pavlovien" (Klima, 1968).

Sur le plan techno-fonctionnel, S.A. Semenov émit l'hypothèse que certaines lames (de Kostienki I) avec des retouches inverses aux deux extrémités auraient été utilisées pour couper par les bords latéraux, ces derniers portant d'ailleurs les traces de ce travail (Semenov, 1973, p. 63). Cette hypothèse, bien que compatible avec l'idée d'amincissement en vue d'un emmanchement, ne fut, semble-t-il, jamais reprise par la suite.

En 1974, M.H. Newcomer et F. Hivernel-Guerre proposèrent une hypothèse interprétative inédite des couteaux de Kostienki I. En s'appuyant sur une comparaison avec des pièces appartenant au Capsien de Gamble's Cave (Kenya), ces deux auteurs pensaient pouvoir considérer ces pièces comme de possibles nucléus à lamelles (Newcomer et Hivernel-Guerre, 1974). Cette réflexion n'était cependant pas étayée par une étude technologique, ce qui l'exposait d'autant plus aisément à la critique. Devant les difficultés à cerner la fonction de ces outils, V.I. Belayeva entreprit en 1977 une nouvelle étude des pièces du site éponyme (Belayeva, 1977). Selon, cet auteur, il n'existait qu'un seul trait distinctif et commun aux pièces du genre: la présence (en partie proximale ou distale) d'une surface de jonction, entre face supérieure et inférieure, aménagée par des retouches inverses. Ces observations la conduisirent donc à considérer qu'il s'agissait d'une technique plutôt que d'un type unique et bien cerné.

Quelques années plus tard, l'hypothèse de M.H. Newcomer et F. Hivernel-Guerre fut réfutée par Marcel Otte qui considérait lui aussi ces aménagements comme des amincissements en vue d'un emmanchement (Otte, 1980, p. 55). J. Kozlowski, s'appuyant sur la définition de V.I. Belayeva, s'opposa lui aussi l'idée que les couteaux de Kostienki du site éponyme puissent être des nucléus à lamelles (Kozlowski, 1984, p. 38). Il redéfinit alors les lames aménagées par technique de Kostienki en précisant la difficulté de les distinguer d'autres groupes typologiques ou d'autres types d'outils. Ses travaux s'appuyaient alors sur une importante série de pièces provenant de Corbiac (Dordogne), apparemment seul gisement de France connu, à l'époque, pour avoir livré des couteaux de Kostienki (Kozlowski, 1984).

Parfois présents en Europe occidentale, les lames aménagées par technique de Kostienki n'en restaient pas moins des éléments plutôt caractéristiques du Gravettien d'Europe centrale ou orientale. K. Valoch, s'attachant à préciser la définition du Pavlovien après $\mathrm{H}$. Delporte et B. Klima, fit ainsi de ces artefacts un élément typologique caractéristique de cette entité gravettienne. Il soulignait toutefois que ces pièces n'étaient pas systématiquement présentes dans les différents sites de référence (Valoch, 1986, p. 55). Au début des années 90, la présence de couteaux de Kostienki était désormais avérée des berges du Don à celles de la Dordogne, mais ces pièces restaient des éléments très anecdotiques au sein du Gravettien français. Ce n'est que récemment qu'une étude technologique approfondie mit en évidence leur présence dans un contexte différent de celui du Sud-Ouest français ${ }^{(1)}$ : les couches gravettiennes du gisement du Blot à Cerzat dans le Massif central en Haute-Loire (fig. 1) (Klaric, 1999).

\section{Problèmes actuels}

La présence discrète mais néanmoins réelle de telles pièces dans des gisements d'Europe occidentale tend à poser la question de leur validité en tant que marqueur typologique spécifique de certains faciès régionaux du Gravettien. Du moins s'avère-t-il nécessaire de bien caractériser les artefacts occidentaux afin de mettre en évidence les similitudes et/ou les différences qui peuvent exister avec ceux provenant d'Europe centrale et orientale. De plus, la multiplicité des interprétations invite à reconsidérer au cas par cas les hypothèses fonctionnelles des pièces qui présentent cette technique d'aménagement. Comme le remarque J. Kozlowski : "Le fait qu'il s'agisse d'une technique spéciale plutôt que d'aspects morphologiques constants, implique qu'il 


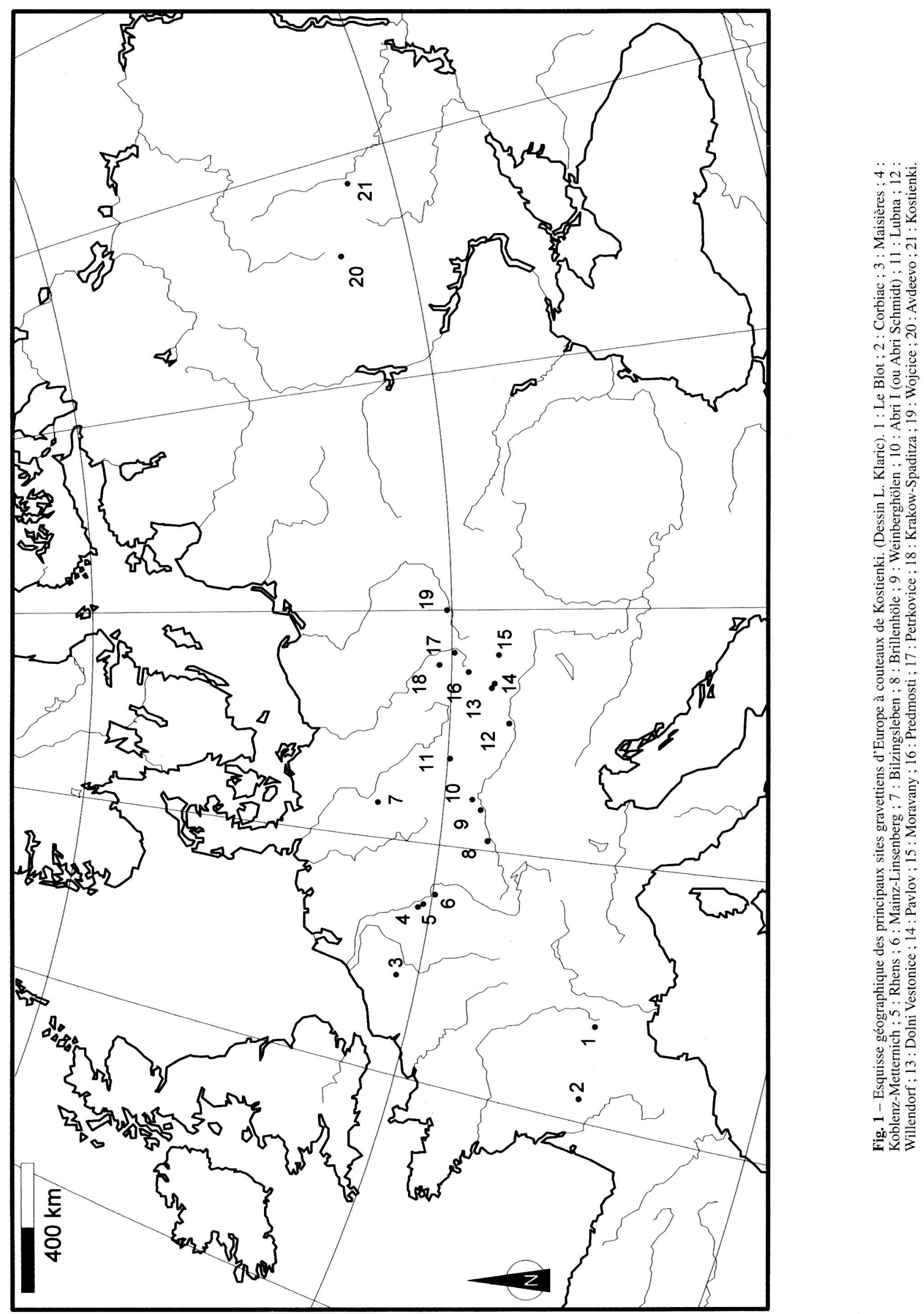


serait difficile d'attribuer une fonction constante et déterminée à ces outils" (Kozlowski, 1984, p. 32).

Il faut par ailleurs souligner l'existence de techniques analogues dans d'autres industries du Paléolithique supérieur (le Magdalénien par exemple). Les pièces résultant de ces techniques ont d'ailleurs connu diverses interprétations, tout comme pour le Gravettien. Ainsi, une démarche technologique permit de démontrer le rôle original de nucléus lamellaires qu' avaient de tels artefacts sur le site Magdalénien du Rocher-de-la-Caille (Loire) (Alix et al., 1995). À l'instar de cet exemple, le gisement du Blot offre une nouvelle opportunité d'interprétation. En effet, une étude technologique approfondie nous permet de considérer que sur ce site, les lames aménagées par technique de Kostienki ont pu jouer le rôle de nucléus (Klaric, 1999).

\section{LES DONNÉES RELATIVES AU GRAVETTIEN DU BLOT \\ Un contexte particulier de production d'armatures microlithiques}

L'ensemble moyen (couches 38 à 44) des niveaux gravettiens du Blot fut attribué au Périgordien VI par D. Buisson (Buisson, 1991). Cet ensemble s'avère particulièrement riche en armatures microlithiques (177 pièces sur environ 243 outils) (fig. 2) ce qui amena ce même auteur à remarquer le point suivant: "Une analyse détaillée des techniques de fabrication des pièces à dos a permis de mettre en évidence l'utilisation fréquente de chutes de burins pour leur confection. Cette observation pose le problème de savoir si les burins du Blot n'ont pas joué le rôle de nucléus dans un contexte où l'économie de matière première était, semble-t-il, de bonne règle. L'absence de nucléus à lames ou lamelles confirmerait cette hypothèse" (Buisson, 1991, p. 107). Ce constat préliminaire guida notre propre étude technologique qui permit de mettre en évidence, au Blot, le rôle de nucléus à lamelles de certains burins (dix-sept en tout) dont plusieurs associés à des aménagements par technique de Kostienki (au moins six) (Klaric, 1999). En effet, en l'absence d'éléments prouvant l'existence d'une production lamellaire sur blocs, il fallut envisager sérieusement l'hypothèse que les chutes de burins lamellaires aient pu être des produits recherchés intentionnellement et non pas seulement le résultat des opérations de façonnage et de ravivage des burins.

Plusieurs éléments accréditent cette hypothèse : d'une part la compatibilité morpho-dimensionnelle des lamelles supports d'armatures et des lamelles de burins. Cette concordance fut démontrée, à la fois par l'examen des négatifs lamellaires présents sur les burins et par une analyse comparative des longueurs, largeurs et épaisseurs des pièces (fig. 3). D'autre part, la présence de certains caractères spécifiques aux lamelles de burins ${ }^{(2)}$ sur plusieurs armatures permet de confirmer l'utilisation très fréquente de tels supports pour la confection des pièces à dos (fig. 2, $\mathrm{n}^{\text {os }}$ 1-5-7-8-12-15). Ajoutons finalement que la lecture des processus technologiques qui conduisirent à l'élaboration de certaines pièces permit de mettre en évidence l'existence d'un schéma original de production de lamelles à partir de morphologies de burins. Au Blot, la production lamellaire s'articule autour de deux modes opératoires distincts mais utilisés presque systématiquement de manière complémentaire : le débitage sur bord de lame (ou "technique du coup de burin") et le "débitage plan" (ou "technique de Kostienki”) (Klaric, 1999).

\section{Particularités des lames aménagées par technique de Kostienki au Blot}

Il n'existe pas au Blot de pièces qui soient seulement des couteaux de Kostienki au sens strict, de même qu'il n'existe aucune "forme intermédiaire" (troncature inverse seule par exemple) comparable à celles identifiées par J. Kozlowski à Corbiac (Kozlowski, 1984). Les aménagements par technique de Kostienki sont systématiquement associés (en partie opposée ou non) à des formes typologiques particulières, notamment des burins (multiple mixte, du Raysse, etc.) (fig. 4). Les six pièces aménagées présentent presque toutes des troncatures inverses et au moins un enlèvement lamellaire plan sur la face supérieure. Il faut toutefois relever une variante de l'aménagement par troncature inverse : certaines pièces présentent un petit enlèvement transversal (assimilable à un coup de burin) qui aménage une surface de plan de frappe propice à l'extraction d'une lamelle plan (fig. $4, \mathrm{n}^{\circ} 3$ ). Détail frappant, les outils auxquels sont associés ces aménagements montrent toujours de nombreux ravivages lamellaires. Autre point remarquable, certains remontages ont permis de mettre en évidence le soin qui fut apporté à la préparation au détachement des lamelles sur la face supérieure (fig. $4, \mathrm{n}^{\circ} 1$ ).

\section{Le "débitage plan": un mode de production lamellaire!}

La production lamellaire à partir des “burins" s'articule, rappelons-le, autour de deux modes opératoires distincts que les tailleurs ont utilisés presque systématiquement de manière combinée. L'extraction d'une lamelle sur une lame-support est possible soit sur le bord (d'un point de vue typologique cela équivaut à la technique du coup de burin), soit sur la face supérieure (et/ou plus rarement inférieure), ce qui correspond à ce que nous appelons le "débitage plan". Afin d'obtenir un plus grand nombre de produits, ces modes opératoires peuvent être employés en association, ce qui conduit à des nucléus présentant diverses morphologies de "burins" très ravivés (Klaric, 1999).

Sans entrer dans le détail de la production lamellaire à partir de telles morphologies, nous souhaitons présenter les modalités propres à l'extraction de lamelles sur la face supérieure d'une lame support, c'est-à-dire le "débitage plan". Notons que le terme de "plan" est ici détourné car il ne s'applique, d'un point de vue typologique, qu'à un enlèvement de coup de burin dont le négatif est "oblique ou presque parallèle à la face d'éclatement (face inférieure) de la pièce qu'il entame largement" (Brézillon, 1977, p. 186). 

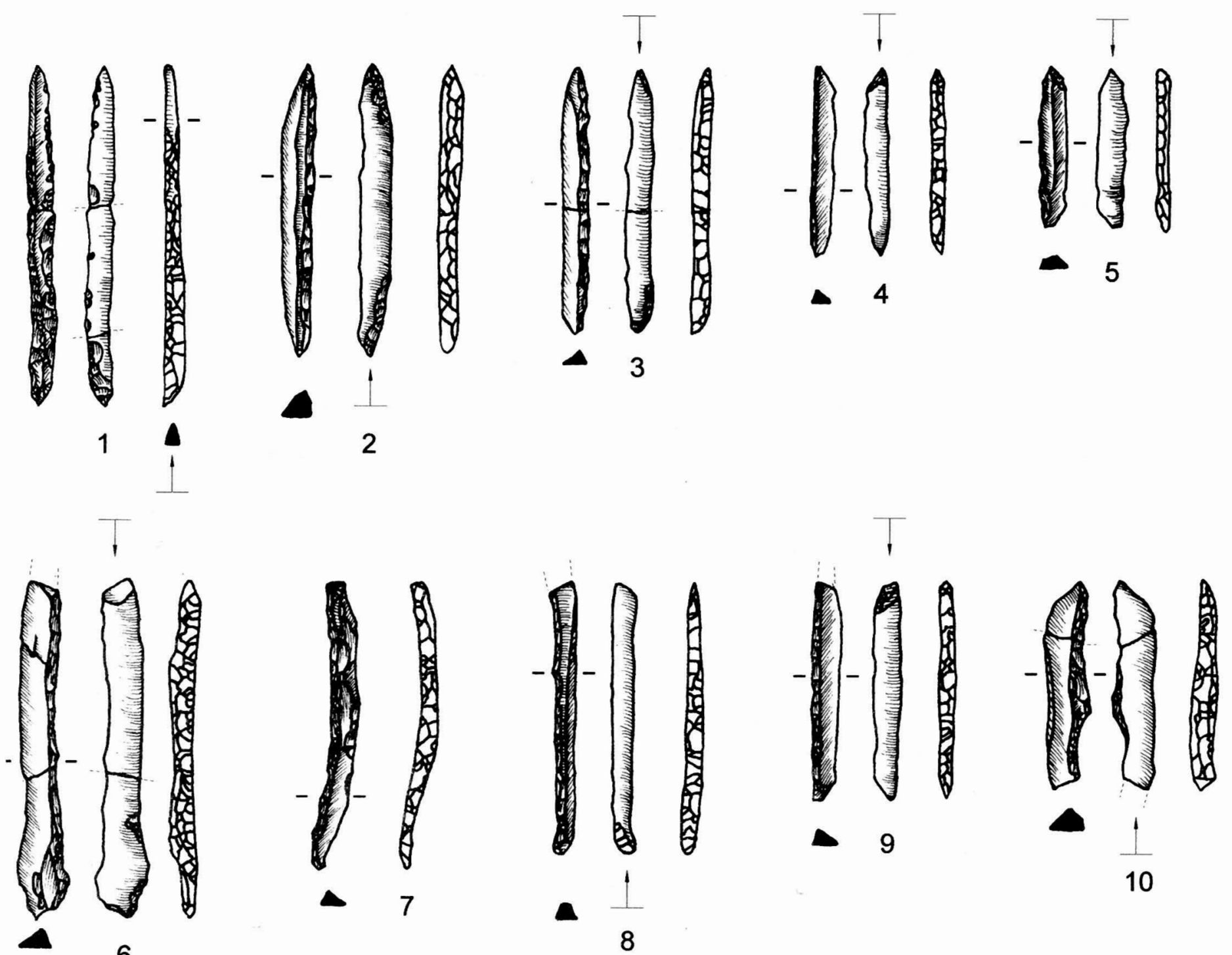

6
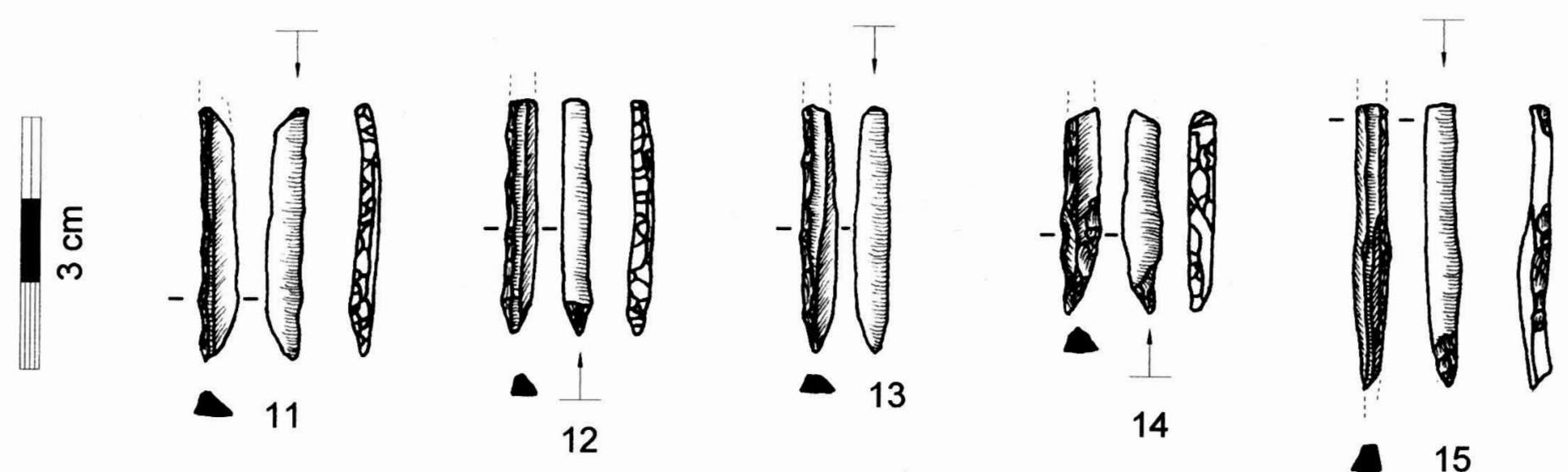

Fig. 2 - Armatures microlithiques gravettiennes de l'ensemble moyen du Blot (Haute-Loire). Les pièces 1-5-7-8-12-15 ont été réalisées sur lamelles de burin. (Dessins L. Klaric). 1 à 4 et 8-9-12-14: microgravettes; 5 à 7 et 10-11-13: lamelles à dos; 15 : pièce à dos cassée en cours de fabrication.

L'objectif d'un tel débitage est l'extraction d'une lamelle qui utilise comme guide une des nervures de la face supérieure d'une lame-support. La section de la lame tient alors lieu de plan de frappe (fig. 5 et 6) et les "flancs" correspondent alors aux bords de la lame. La face inférieure constitue donc une sorte de "dos en méplat". Le recul paraît ainsi relativement faible puisqu'il se développe dans la partie la moins épaisse de la 
Histogramme comparatif des classes de longueurs des lamelles de burins brutes entières (effectif: 120) et des armatures entières (eff : 9)

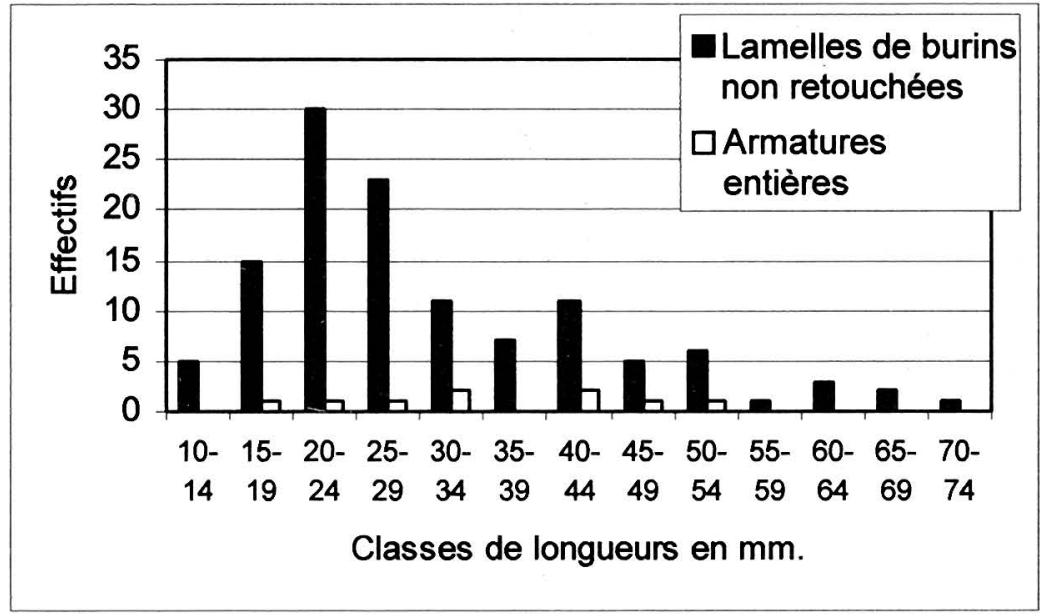

Histogramme comparatif des largeurs de lamelles de burins brutes entières et fragmentées (eff : 364 ) et de toutes les armatures (eff : 176)

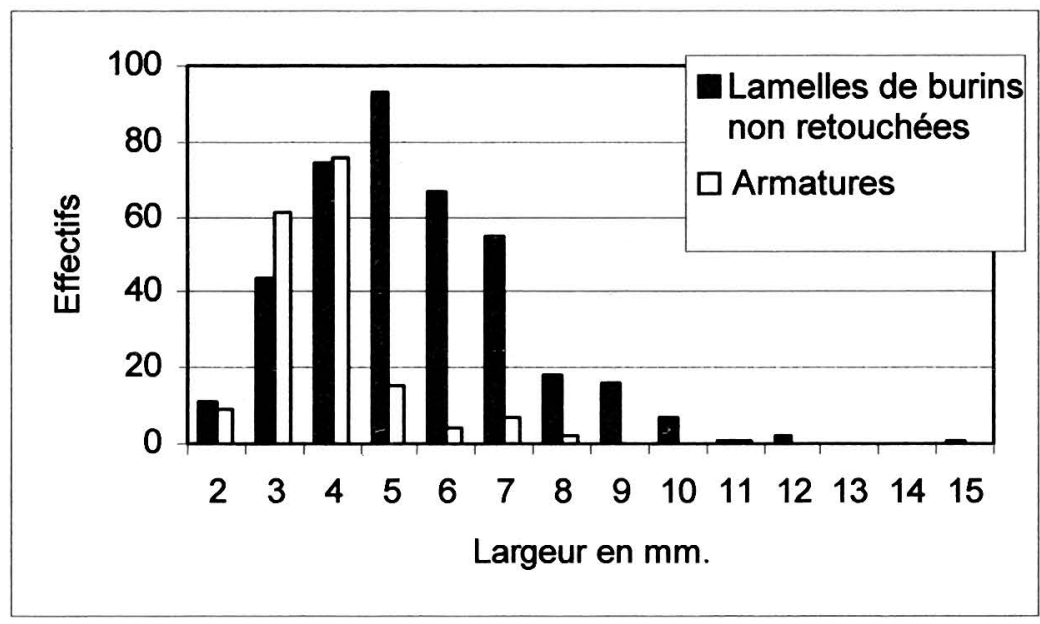

Histogramme comparatif des épaiseurs de lamelles de burins brutes entières et fragmentées (eff : 364) et de toutes les armatures (eff : 176)

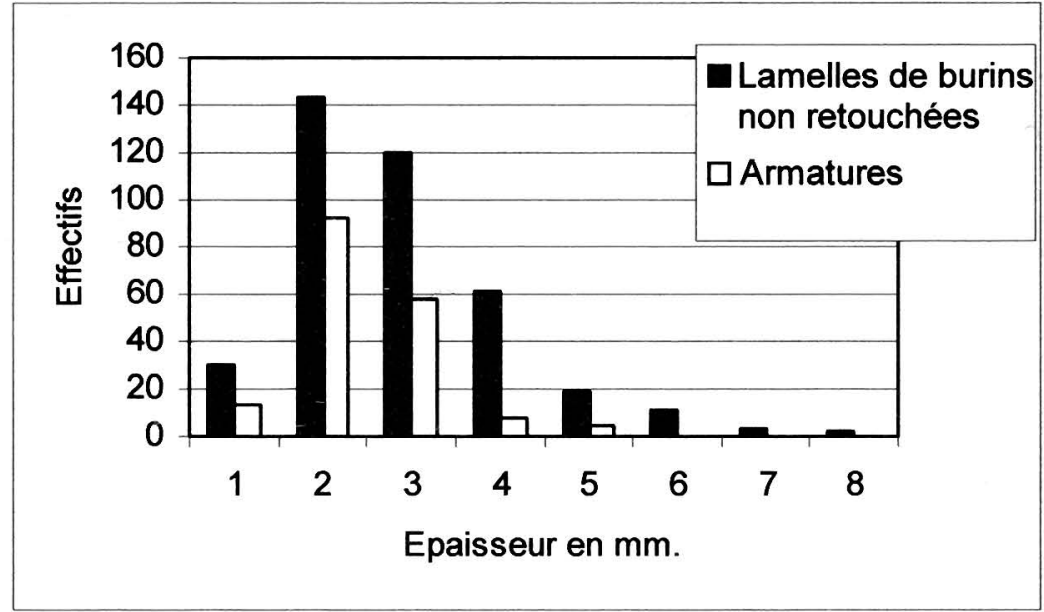

Fig. 3 - Histogrammes comparatifs des données dimensionnelles des lamelles de burins brutes et des armatures de l'ensemble moyen du Blot. 

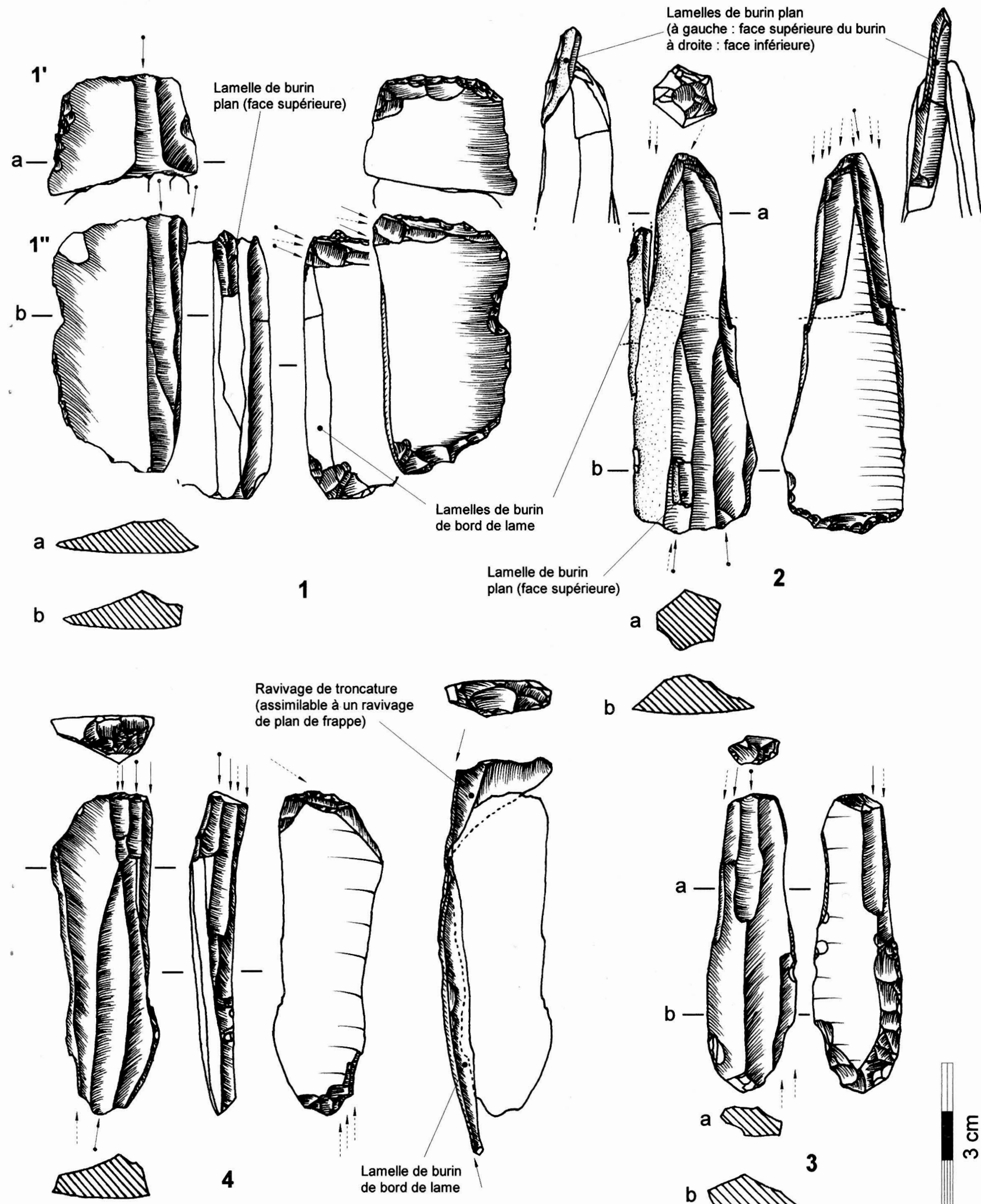

3

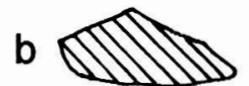

Fig. 4 - Burins sur lames associés à des aménagements par technique de Kostienki (ensemble moyen), le Blot. (Dessins D. Buisson et L. Klaric). 1 : lame aménagée par technique de Kostienki (cf. fig. 6) ; 1': fragment de lame aménagée par technique de Kostienki ; 1" : burin d'angle sur cassure associé à un aménagement par technique de Kostienki ; 2 : burin dièdre déjeté prismatique associé à un aménagement par technique de Kostienki en partie opposée ; 3 : burin du Raysse associé à un aménagement par technique de Kostienki ; 4 : burin multiple mixte associé à un aménagement par technique de Kostienki. 


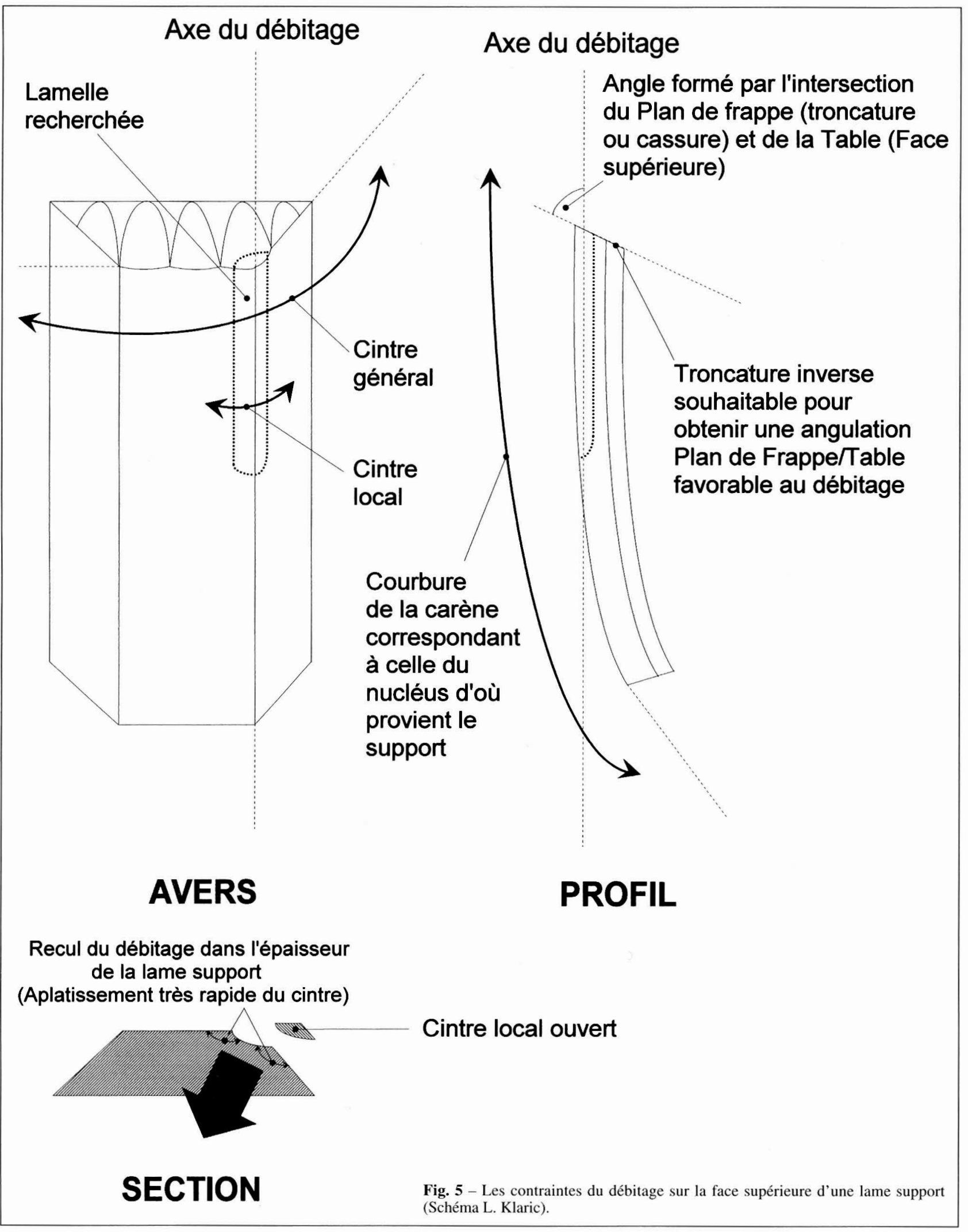

lame-support (fig. 5). Néanmoins, l'avantage de ce principe de débitage est un positionnement dans le volume qui confère à la table lamellaire (la face supérieure) des qualités de cintre et de carène relativement favorables à l'extraction des produits (fig. 5).

Il convient de distinguer le cintre général (qui correspond à celui de la face supérieure de la lame-support) du cintre local qui correspond lui à l'angle formé par la nervure guide sur laquelle s'appuie la lamelle recherchée. Pour que le cintre local soit favorable à l'extraction d'un produit, il est préférable que son angulation ne soit pas trop largement ouverte. Cela contraint donc le tailleur à choisir une lame plutôt épaisse où le cintre d'au moins une des nervures est sensiblement inférieur 

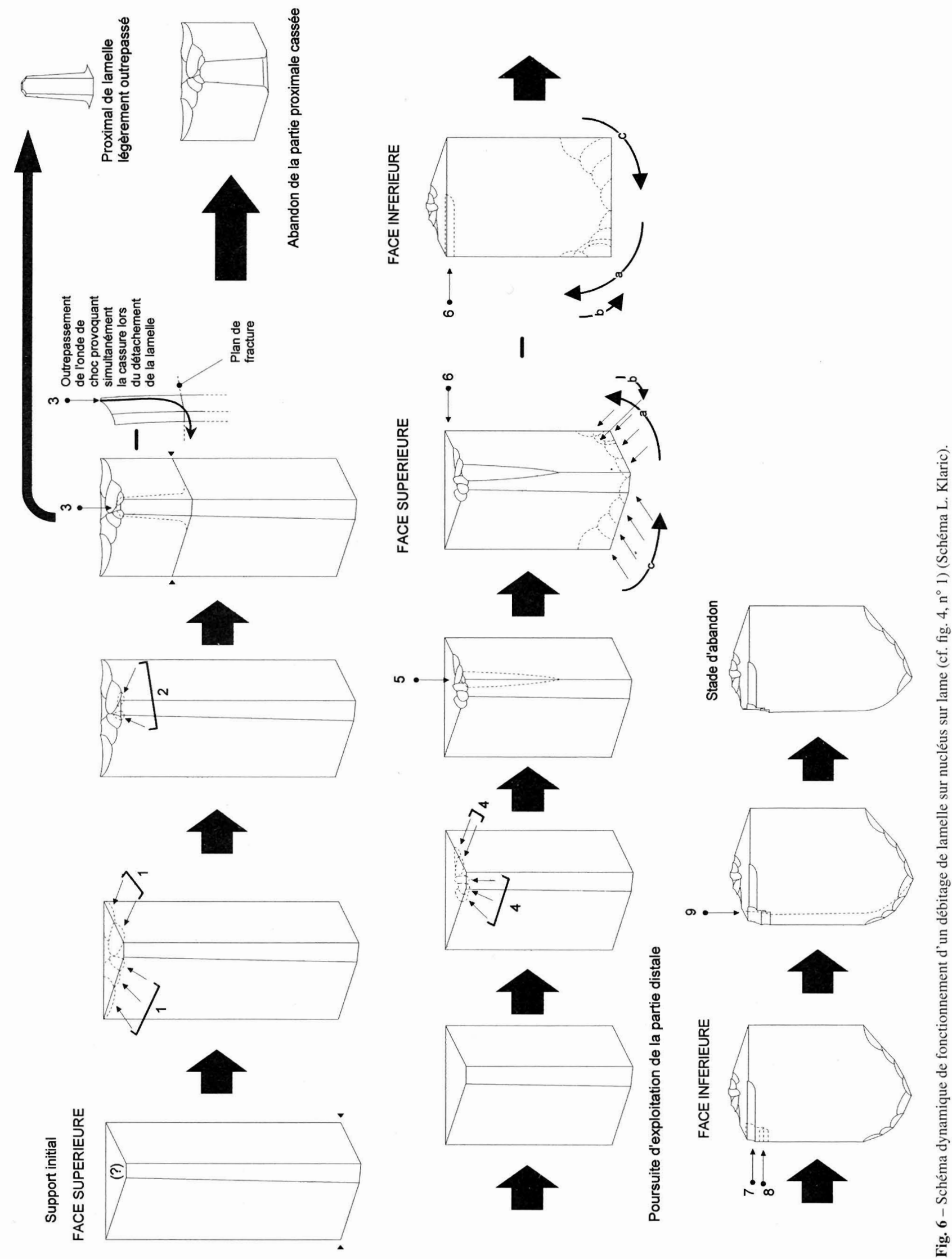


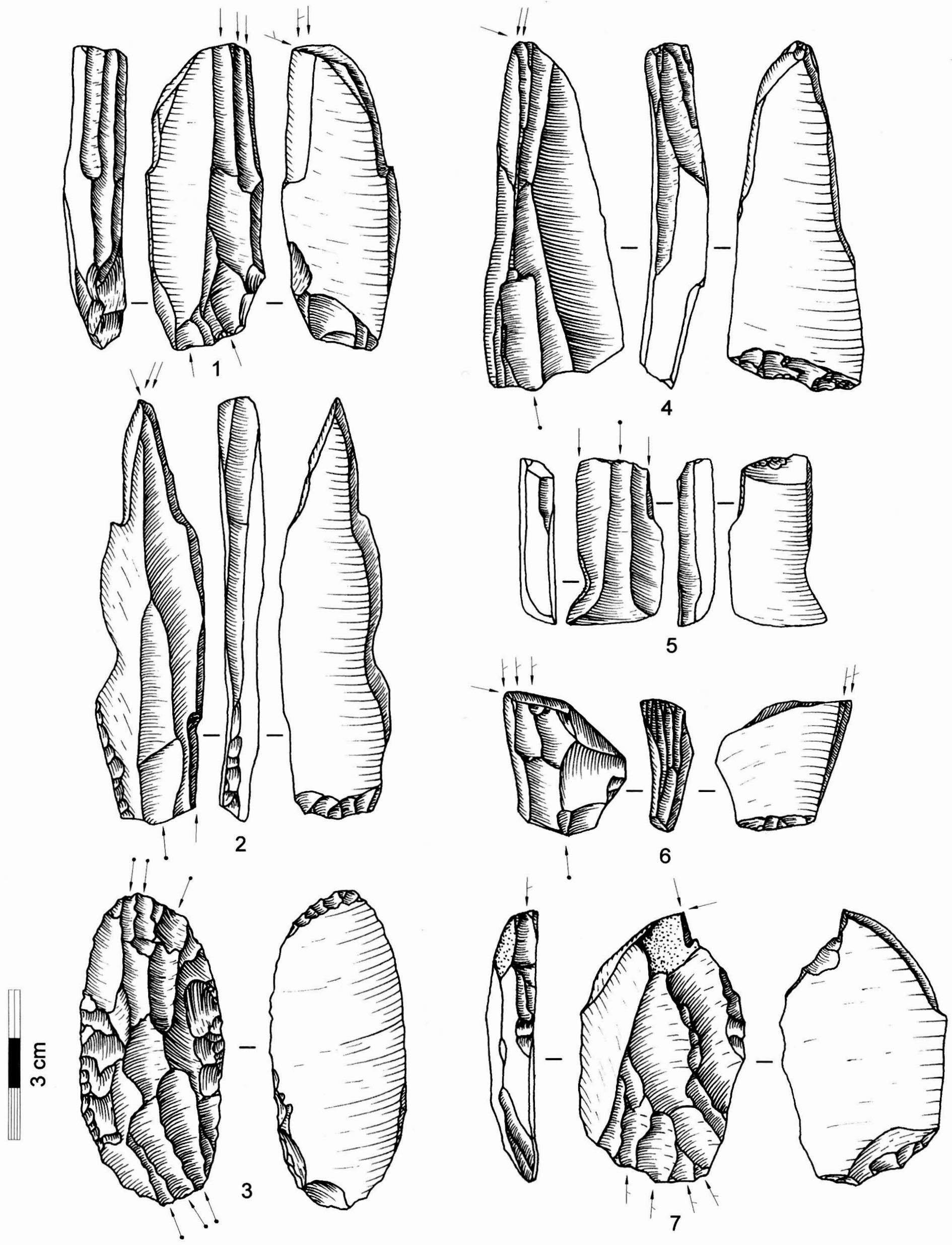

Fig. 7 -Différents types de couteaux de Kostienki. (Dessins L. Klaric d'après références). 1 : burin polyédrique associé à un aménagement par technique de Kostienki (Willendorf II, niveau 9) (d'après Otte, 1981) ; 2 : burin dièdre associé à un aménagement par technique de Kostienki (Willendorf I) (d'après Otte, 1981) ; 3 : couteaux de Kostienki (Kostienki I) (d'après Efimienko, 1958) ; 4 : burin polyédrique associé à un aménagement par technique de Kostienki (Mainz-Linsenberg) (d'après Hahn, 1969) ; 5 : burin d'angle associé à un aménagement par technique de Kostienki (Mainz-Linsenberg) (d'après Hahn, 1969); 6 : burin polyédrique associé à un aménagement par technique de Kostienki (Brillenhöle, niveau 7 ) (d'après Otte, 1981); $7:$ burin dièdre associé à un aménagement par technique de Kostienki (Pavlov II) (d'après Otte, 1981). 
à $180^{\circ}$ mais apparemment toujours largement supérieur à $90^{\circ}$. Ainsi, le cintre local de la nervure guide est alors raisonnablement ouvert, sans pour autant être trop plat (fig. 5). Cette angulation, bien que propice au débitage, implique néanmoins un risque permanent de réfléchissement ou d'étalement ${ }^{(3)}$ de l'onde de fracture si la force appliquée au détachement de la lamelle est mal appréciée.

En revanche, l'installation de la table lamellaire sur la face supérieure semble être une solution d'emblée très favorable en terme de carène. La lamelle recherchée exploitant comme guide une des nervures de la lamesupport, elle bénéficie d'un carénage identique à celui de cette même lame (fig. 5). Dans la plupart des cas, la face supérieure d'un support laminaire est plutôt légèrement convexe, ce qui lui confère des propriétés de carène favorable à un débitage. Il n'est donc pas nécessaire de procéder à des opérations de taille dont le but serait d'améliorer ou de corriger le carénage. Cependant, pour que l'extraction de la lamelle soit possible, il faut que la surface débitée soit suffisamment régulière et ne comporte pas d'éléments perturbateurs (inclusions diverses, anciens réfléchissements, fractures de gel, etc.).

La dernière contrainte s'appliquant à ce type de débitage concerne l'angulation entre la surface débitée (face supérieure) et la surface de plan de frappe (troncature inverse ou cassure). Il est absolument nécessaire que l'angulation entre ces deux surfaces soit sensiblement inférieure à $90^{\circ}$ (voire proche de $45^{\circ}$ ). Si cette condition n'est pas remplie, deux types d'accidents sont à redouter: le réfléchissement en partie proximale de table ou l'écrasement du plan de frappe sans que la lamelle soit extraite. Les tailleurs semblent avoir résolu cette difficulté soit par l'aménagement d'une troncature inverse très anguleuse (fig. $4, \mathbf{n}^{\circ} 4$ ), soit par l'aménagement d'un talon proéminent en "micro-éperon" (par surcreusements latéraux du plan de frappe) (fig. $4, \mathrm{n}^{\circ} 1$ et fig. $\left.6, \mathrm{n}^{\circ} 4\right)$.

Que la section de la lame-support soit triangulaire ou trapézoïdale, l'extraction d'une lamelle conduit toujours un aplatissement très rapide du cintre, ce qui a pour conséquence une faible productivité. La récurrence de ce type de débitage n'est donc pas très développée. Le tailleur est donc obligé de passer à l'exploitation d'un autre secteur de lame-support s'il veut poursuivre son débitage. Cela explique que cette modalité de débitage se combine presque systématiquement avec une autre modalité d'extraction de lamelles (le débitage sur bord de lame ou "technique de coup de burin") ce qui aboutit, répétons-le, à des nucléus sur lames aux morphologies de "burins très ravivés" (Klaric, 1999). Nous n'avons présenté ici que le débitage plan sur la face supérieure d'une lame-support, mais il existe une variante plus rare qui consiste en l'extraction d'une lamelle sur la face inférieure. Cette variante obéit bien sûr à des contraintes particulières et n'apparaît jamais de manière autonome (Klaric, 1999).

\section{CONCLUSION}

Le principal objectif de cet article est de signaler l'existence des lames aménagées par technique de Kostienki dans certains ensembles du Gravettien français. Il s'agit d'attirer l'attention des chercheurs sur le rôle possible de nucléus de ces pièces, du moins dans un contexte particulier de production d'armatures microlithiques. L'ambition est également de mieux caractériser cette catégorie typologique qui, malgré de nombreuses tentatives, répond à des définitions typo-fonctionelles trop vagues. Aussi s'avère-t-il nécessaire d'étudier les différentes formes de ces aménagements (fig. 7) et de redéfinir éventuellement des types distincts plus pertinents. Dans le cas du Blot, c'est l'approche technologique qui a permis une redéfinition de ce genre de pièce. Des approches similaires seraient à envisager pour certains sites d'Europe centrale et orientale riches en couteaux de Kostienki (fig. 1). Ces nouvelles réflexions permettraient alors de reconsidérer la question de l'unité fonctionnelle de ce type souvent considéré comme marqueur chrono-culturel. Cela aboutirait peut-être à terme à une meilleure caractérisation de certains faciès régionaux du Gravettien.

Remerciements : je tiens à remercier monsieur Henri Delporte qui m'a offert la possibilité d'étudier le Gravettien du Blot et qui m'a autorisé à publier cet article. Merci également à Boris Valentin pour ses relectures attentives et ses conseils avisés.

\section{Notes}

(1) Cette remarque ne tient pas compte d'un article récemment paru dans lequel il est précisé que des couteaux de Kostienki ont été identifiés à la Grotte du Marronnier, à Saint-Remèze sur la rive gauche de l'Ardèche (Onoratini et al., 1999, p. 159-160). Je tiens donc à m'excuser auprès des auteurs de cet article puisqu'il ne m'a pas été possible d'inclure les données issues de leurs travaux. Il sera néanmoins très intéressant de comparer les résultats de leur analyse des couteaux de Kostienki (en cours) avec l'approche développée dans le présent article. En revanche les auteurs signalent aussi la présence de couteaux de Kostienki dans le gisement de la Vigne-Brun (Loire) (Onoratini et al., 1999, p. 160). Il me semble toutefois que cela n'est attesté par aucune publication explicite. En l'absence d'élément plus concret, je n'ai donc pas souhaité inclure ce site à ma réflexion.

(2) Ces éléments sont entre autres : la présence d'un "pan-revers" (pan d'une lamelle constitué par une partie de la face inférieure du support dont elle est extraite (Le Mignot, 1998 ; Klaric, 1999, p. 19), la présence d'un bord retouché antérieurement à l'extraction, l'existence de négatifs antérieurs burinants, la rectitude du support, le caractère rebroussé du support en partie distale, etc.

(3) Dispersion de l'onde de fracture dans la matière mais qui ne produit pas de réfléchissement. 


\section{RÉFÉRENCES BIBLIOGRAPHIQUES}

ALIX P., PELEGRIN J., DELOGE H. (1995) - Un débitage original de lamelles par pression au Magdalénien du Rocher-de-la-Caille (Loire, France), Paléo, n 7 , p. 187-199.

BELAYEVA V.I. (1977) - Opyt sozdaniya metodiki opisanya " nojey kostenkovoskogo tipa". Problemy paleolita vostotchnoy i centralnoy Evropy. Moscou.

BREUIL H. (1924) - Notes de voyage paléolithique en Europe centrale. L'Anthropologie, XXXIV, Paris, p. 515-533.

BRÉZILLON M. (1977) - La dénomination des objets en pierre taillée. Matériaux pour un vocabulaire des préhistoriens de langue française, $\mathrm{IV}^{\mathrm{e}}$ supplément à Gallia Préhistoire, Paris, éd. du CNRS, 423 p.

BUISSON D. (1991) - Le Périgordien du Blot, Bulletin de la Société Préhistorique Française, t. 88, p. 104-108.

\section{EFIMIENKO P.P. (1958) - Kostienki I, Kiev.}

GVOZDOVER M.D. (1961) - Spetsifitchesikiye tcherty kamennogo inventaria Avdeevskoy paleolitcheskoy stoyanki, Moscou, kratkiye soobchtenia Instituta Arkheologui, vol. 82.

HAHN J. (1969) - Gravettien-Freilandstationen im Rheinland : MainzLinsenberg, Koblenz-Metternich und Rhens, Bonner Jahrbücher, t 169 , p. 44-87.

HEINZELIN J. de (1962) - Manuel de typologie des industries lithiques. Bruxelles, 74 p.

HEINZELIN J. de (1973) - L'industrie du site paléolithique de Maisières-Canal. Bruxelles, Institut Royal des Sciences Naturelles, Mémoire n $171,63 \mathrm{p}$.

KLARIC L. (1999) - Un schéma de production lamellaire original dans l'industrie gravettienne de l'ensemble moyen du gisement du Blot à Cerzat (Haute-Loire), Mémoire de DEA de l'Université de Paris I, 2 vol., 64 p.

KLIMA B. (1968) - Das Pavlovien in den Weinberghölen von Mauern, Quartär, t. 19, p. 263-273.
KOSLOWSKI J.K. (1984) - Les lames aménagées par la "technique Kostienki" dans le Périgordien supérieur de Corbiac, in : "Advances in Paleolithic and Mesolithic Archaeology", Archaeologia interregionalis, Varsovie, p. 31-78.

LE MIGNOT Y. (1998) - La question de la production d'armatures sur le site Gravettien de Plasenn-Al-Lomm (Côtes d'Armor), Mémoire de DEA de l'Université de Paris I, $60 \mathrm{p}$.

NEWCOMER M.H., HIVERNEL-GUERRE F. (1974) - Nucléus sur éclat : technologie et utilisation par différentes cultures préhistoriques, Bulletin de la Société Préhistorique Française, t. 71, p. 119-128.

ONORATINI G., CHAREYRE R., COMBIER J., DAL-PRA G., THINON M. (1999) - Nouvelles données sur la culture gravettienne en Vivarais, in: Les faciès leptolithiques du nord-ouest méditerranéen : milieux naturels et culturels, XXIV Congrès Préhistorique de France, Carcassonne 26-30 septembre 1994, p. 151-163.

OTTE M. (1979) - Le Paléolithique supérieur ancien de Belgique. Bruxelles, Musées Royaux d'arts et d'Histoire, 684 p.

OTTE M. (1980) - Le "couteau de Kostienki", Helinium, t. 20, p. 54-58.

OTTE M. (1981) - Le Gravettien en Europe centrale. Brugge, De Tempel, Dissertationes Archaeologicae Gandenses, 2 vol.

SEMENOV S. A. (1973) - Prehistoric Technology. London, Cory, Adams and Mackay, $211 \mathrm{p}$.

VALOCH K. (1986-1987) - Les questions du Pavlovien, Antiquités Nationales, t. 18-19, p. 55-62.

\section{Laurent KLARIC}

Doctorant de l'Université de Paris I Laboratoire d'Ethnologie Préhistorique - E.P. 1730 Maison de l'Archéologie 21, allée de l'Université, F-92023 Nanterre Cedex 